Dr. med. Dirk Einecke Chefredakteur dirk.einecke@springer.com

\title{
Herz-Kreislauf-Erkrankungen \\ Drei Mega-Studien, die Sie kennen sollten
}

\begin{abstract}
Auf der Jahrestagung der Europäischen Kardiologie-Gesellschaft (Barcelona, 26.30.8.2017) wurden die Ergebnisse von drei besonders großen Interventionsstudien vorgestellt, die man kennen sollte:
\end{abstract}

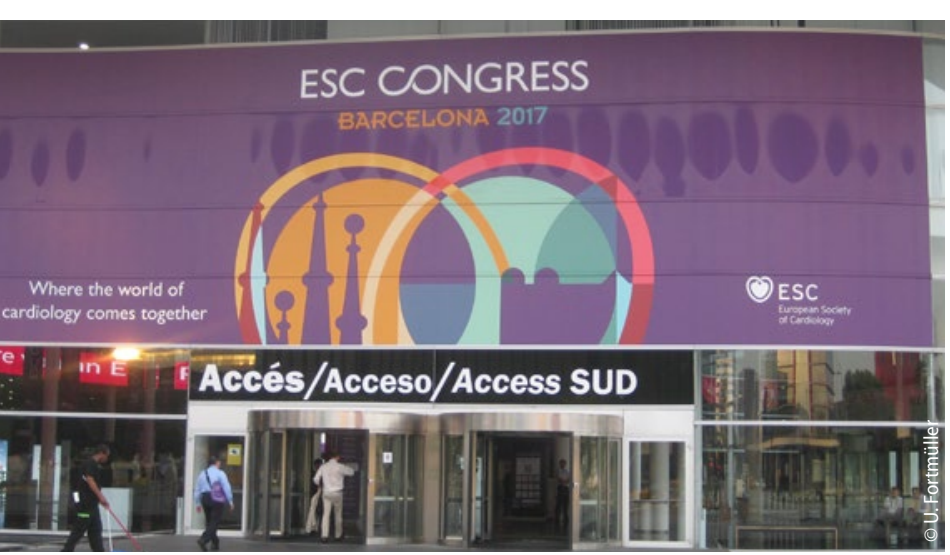

1. Die COMPASS-Studie. Bei 27.395 Patienten mit KHK oder PAVK wurden Sekundärpräventions-Strategien verglichen. Rivaroxaban (Xarelto ${ }^{\circ}, 2$ x 2,5 mg/d) plus ASS schützte über 23 Monate besser vor Herz-Kreislauf-Tod, Infarkt und Schlaganfall als ASS allein $(4,1 \%$ vs. $5,9 \%$ ), auf Kosten eines höheren Risikos beherrschbarer Blutungen $(3,1 \%$ vs. $1,9 \%)$. Wird sich die Low-dose-Antikoagulation bei KHK etablieren? 2. Die CANTOS-Studie. Bei 10.061 HochrisikoPostinfarkt-Patienten mit erhöhten Entzün-

\section{Was Kardiologen sonst noch so erforschen \\ Nützliches für die Hausarztmedizin}

\section{Heiraten schützt das Herz, vor allem verheiratet bleiben!}

Verheiratete haben gegenüber Alleinstehenden einen signifikanten Gesundheitsvorteil, wenn sie im Alter eine Herzkrankeit ereilt, etwa in Gestalt eines akuten Koronarsyndroms. Einer sehr großen Untersuchung aus England zufolge (ACALMStudie) liegt die Wahrscheinlichkeit um $14 \%$ höher, das Ereignis zu überleben.

\section{Fett essen verlängert das Leben}

Endlich engagiert sich die Forschung einmal gegen kulinarische Grausamkeiten. Schon lange ist bekannt, dass die Reduktion des Fettanteils in der Nahrung den Geschmack minimiert. Nun erfahren wir aber: Wer mehr Fett verzehrt, lebt signifikant län- ger als derjenige, der sich diesbezüglich kasteit. Reduziert werden sollten die Kohlenhydrate - eine hohe Zufuhr erhöht das Sterberisiko um 28\%. Dies ist ein Ergebnis der PURE-Studie mit 135.000 Teilnehmern.

\section{Gefäße offen halten mit Schokolade und Olivenöl}

Liebhaber sanfter Prävention wissen es schon längst: Dunkle Schokolade, reich an Flavonoiden, hat protektive Effekte auf die von vielen Stressoren des Alltags malträtierten Gefäßwände - regelmäßiger Verzehr vorausgesetzt. Nun wurde eine Studie präsentiert, der zufolge ein signifikanter Anstieg endothelialer Progenitorzellen erreicht wird, wenn die dunkle Schokolade mit $10 \%$ igem nativen Olivenöl angereichert ist. dungswerten (hsCRP $>2 \mathrm{mg} / \mathrm{l}$ ) konnte erstmals gezeigt werden, dass ein antientzündliches Medikament (Canakinumab, Ilaris ${ }^{\circ}$ ) das Risiko für schwere kardiovaskuläre Komplikationen reduziert. Experten sehen einen Durchbruch für die antientzündliche Therapie bei KHK, wenn auch nicht unbedingt mit dem hier untersuchten Medikament.

3. Die REVEAL-Studie. Bei 30.449 Patienten mit atherosklerotischen Gefäßerkrankungen wurde der Wert eines neuartigen Lipidsenkers untersucht. Obwohl alle Studienpatienten mit Atorvastatin schon exzellent eingestellt waren (LDL im Schnitt $61 \mathrm{mg} / \mathrm{dl}$ ), reduzierte der CETP-Hemmer Anacetrapib das Risiko für kardiovaskuläre Komplikationen im 4-Jahresverlauf von $11,8 \%$ auf $10,8 \%$. Der Effekt ist moderat, aber signifikant. Spannend wird die Frage, für welche Patienten dieser Lipidsenker indiziert sein wird.

Man spürt förmlich, wie einem das Herz aufgeht, wenn sich diese Progenitorzellen im Blut vermehren, um für eine reibungslose Endothelfunktion zu sorgen!

Angeboten wird die Schokoladenvariante von einem Studiensponsor aus Arezzo als „Toscolata Olio“ für 11 Euro die Tafel. Wer sie beim Hersteller vor Ort ersteht, darf vermutlich mit noch weiteren kardioprotektiven Glücksgefühlen rechnen.

Ausführliche Berichte über diese Studien

... und andere Neuigkeiten vom europäischen Herzkongress lesen Sie in der nächsten Ausgabe der MMW oder auf dem Portal von Springer Medizin unter: $\rightarrow$ www.springermedizin.de/esc-2017/ esc-2017/14222146. 